\title{
Design of Terahertz-Wave Transmission Imaging System
}

\section{Pei Jie}

The Department of Aeronautics Ammunition Engineer The First Aeronautic Institute of Air Force

Xinyang, China

pei.jie@163.com

\author{
Chen Gang \\ The School of Information Science and Engineering \\ Southeast University \\ Nanjing, China \\ chengang5297602@yahoo.com.cn
}

\begin{abstract}
An imaging system based on the transmission and reflection modes in the terahertz (THz) region is developed by using a backward-wave oscillator (BWO) as source, a GolayCell as detector, and an oscilloscope as data acquisition unit. The system software based on the oscilloscope is designed to control the object movement, and the capture and display of the continuous THz wave image data. The imaging of different objects is tested at the frequencies of $450 \mathrm{GHz}$ and $890 \mathrm{GHz}$ to show the validity of the imaging system at room temperature. The influence of object's humidity and thickness, incident wavelength, and translation step on the $\mathrm{THz}$ imaging is discussed in details.
\end{abstract}

Keywords-Terahertz imaging; backward-wave oscillator; oscilloscope; continuous wave; transmission imaging

\section{INTRODUCTION}

Terahertz (THz) radiation is considered as the electromagnetic spectrum whose frequency ranges from 0.1 $\mathrm{THz}$ to $10 \mathrm{THz}$, lying between the microwave and infrared regions. This part of the electromagnetic spectrum remains the least explored region mainly due to the technical difficulties involved in making efficient and compact $\mathrm{THz}$ sources and detectors. The lack of suitable technologies led to the $\mathrm{THz}$ band being called as the "THz gap" [1]. This technical gap has been rapidly diminishing for the last two decades [2]. A lot of research results demonstrated that $\mathrm{THz}$ waves can penetrate many materials and generate images with high spatial resolution [1], such as $\mathrm{THz}$ radar [3], scattering [4], security detection [5, 6], tomography [7], biology and medicine [8].

$\mathrm{THz}$ imaging technologies can be divided into coherent and incoherent. The coherent technology mainly includes the time- domain spectroscopy imaging, electro-optic sampling imaging, and tomography. While the incoherent technology is mainly the continuous-wave (CW) imaging [9-11]. Most of the initiative work on $\mathrm{THz}$ imaging has utilized timedomain measurements [12], whose advantage is the vast information due to broadband operation, providing good resolution of imaging. However, the low intensity limits their applications due to the long time to scan objects. Recently there also exists $\mathrm{THz}$ imaging using $\mathrm{CW} \mathrm{THz}$ radiation sources such as the frequency-multiplied Gunn diode sources [13], the backward-wave oscillator (BWO) [14], photomixing [15], and quantum cascade lasers [16], because they can provide higher output power and frequency stability for better signal-to-noise ratio. However, CW has a narrow spectral line width compared to the pulsed source. Generally,
CW imaging is more compact and less expensive, and hence it has emerged as an alternative to the pulsed $\mathrm{THz}$ imaging.

In this paper, a compact CW imaging system is built up. The details of the imaging system setup based on the oscilloscope are presented, including hardware and software. Examples of various applications are provided to demonstrate the potential applications of the imaging system.

\section{EXPERIMENTAL SYSTEM}

\section{A. Basic Imaging Principle}

The basic principle of $\mathrm{THz}$ imaging is simply based on the fact that the electric-field intensity distribution is affected by the $\mathrm{THz}$ scattering caused by transmission and reflection properties of the object, including internal defects or damage to the edge of the object. Thus the THz images of the object will display different luminance distributions, which can be used to infer the position of internal defects or damage inside the object and reflect the different characteristics of the object surface.

\section{B. Experimental Setup}

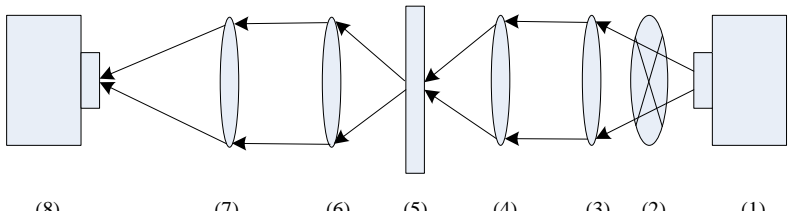

(8)

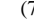

(6)

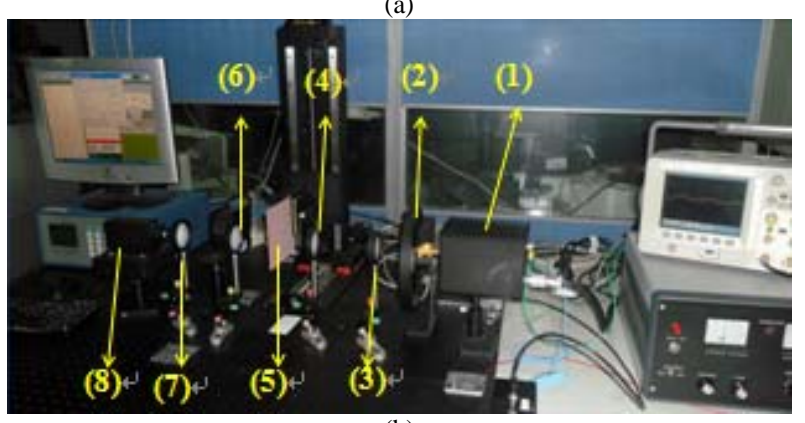

(b)

Figure 1. (a) The schematic diagram of the transmission imaging system, in which (1) is BWO source, (2) chopper, (3) lens with focal length 120 $\mathrm{mm}$, (4) lens with focal length $60 \mathrm{~mm}$, (5) object on a two-dimensional electronically controlled translation stage, (6) lens with focal length 120 $\mathrm{mm}$, (7) lens with focal length $60 \mathrm{~mm}$, and (8) Golay-Cell detector and oscilloscope. (b) The photograph of the transmission imaging system. 
The experimental platform consists of hardware and software. The schematic and experimental setup of transmission imaging systems is demonstrated in Fig. 1.

The THz source is a BWO produced by the Microtech Instruments Inc. (Model QS2-180). The beam from BWO is chopped by a chopper at the rate of approximately $14 \mathrm{~Hz}$. The lenses are made of polythene. A two-dimensional (2D) electronically controlled translation stage consists of a servo motor controller and linear translation stage with the maximum resolution of $0.00032 \mathrm{~mm}$ and maximum velocity of $40 \mathrm{~mm} / \mathrm{s}$. The beam splitter is produced by Russian Tydex Company, with a diameter of $50.8 \mathrm{~mm}$ and thickness of 3.5 $\mathrm{mm}$. The material of the beam splitter is high resistivity float zone silicon (HRFZ-Si). The $\mathrm{THz}$ detector is Golay-Cell from Microtech Instruments Inc. The object is scanned by moving the object under the fixed $\mathrm{THz}$ source and detector. The scanning operation system consists of linear and rotary translation stages, which are controlled by computer through the serial port. The data form the Local Area Network (LAN) of oscilloscope (Agilent DSO5052A) are acquired and stored by computer.

According to the principle of thermal expansion, GolayCell detector can work at room temperature. Its sensitivity is higher than the pyroelectric detector, with the disadvantage of being more sensitive to vibration. Golay-Cell is also sensitive to the infrared flux in ambient, which adds noise to the measurement. In the long process of signal detection, the energy accumulation in the detector can cause Direct Current (DC) drift of the detected signal and reduce the accuracy of detector. To avoid the occurrence of the above circumstances, use a chopper to make the continuous waves from the $\mathrm{THz}$ source be cut into alternating signal. Thus the bandwidth is decreased to reduce the noise and eliminate the $1 / \mathrm{f}$ noise (the DC drift of detector). The signal power can be obtained by measuring the peak to peak value of the alternating signal sent by the detector.

The object fixed on the translation stages is placed at the focus of $\mathrm{THz}$ beam for the maximum spatial resolution, and is scanned along the $x-y$ plane perpendicular to the incident direction with the " $\mathrm{S}$ " type scanning. Then the transmission signal or the reflection signal is obtained by the Golay-Cell detector. The signal from the detector is finally acquired by the oscilloscope. The automatic control process and data recorded by the oscilloscope are controlled by software designed for the imaging system.

\section{Software Design}

The system software is designed using the Microsoft Foundation Class (MFC) library in Microsoft Visual C++ 6.0 Using the structure based on the type of dialog box, the software interface is shown in Fig. 2. The software is mainly composed of three modules, including the platform operation control module, the serial data processing module based on the Electronic Industry Association-Recommended standard232 (RS-232), and the LAN interface data acquisition module.

As shown in Fig. 2, "Current Position" displays the current position of the linear translation stage and rotary translation stage. "Coordinate Reset" means the reset operation of the chosen coordinate axis. "Zero Setting" controls the linear translation stage from the current position to terminal position. "Positive Zero" moves to the terminal and "Negative Zero" moves to the motor terminal of the linear translation stage. "Starting Point" means the starting position of the object in the $\mathrm{x}$ - and $\mathrm{y}$-axis. "End Point" means the ending position of the object in the $\mathrm{x}$ - and $\mathrm{y}$-axis. "Single Step Interval" sets the moving distance of each step. "Time Delay" sets the pause time of each step so as to facilitate manual observation. The default value of the time delay is 0 . When the object needs to be scanned along one direction, can click "X Single-line Scanning" button or "Y Single-row Scanning" button after setting the corresponding parameter. When the object is required to move only one step along one direction, "X Single-step Motion" button or "Y Single-step Motion" button can be clicked after setting the single step interval and velocity. The "Circular Motion" button can be clicked to make the object move along the horizontal direction (x-axis) and vertical direction (y-axis). The program controls the line translation stage to move along the $\mathrm{x}$-axis first and then along the $\mathrm{y}$-axis, thus forms the " $\mathrm{S}$ " type auto-scanning modes.

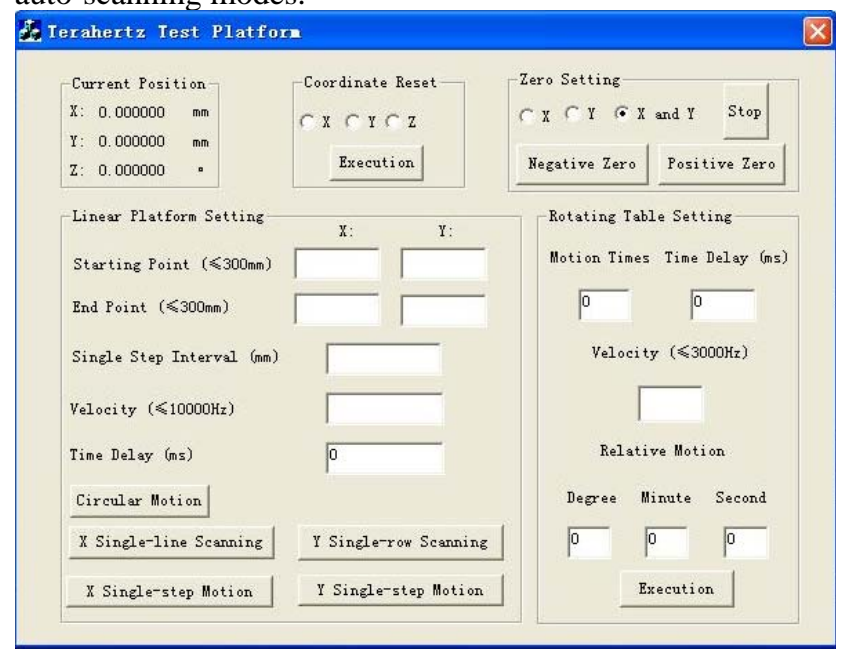

Figure 2. The software interface of the THz imaging platform.

The precise motion of the translation stage is controlled by RS-232 serial communication between the computer and the controller. The serial communication code is completed by Microsoft Communication Control (MSComm) offered by the Windows platform. The code is accomplished by three kinds of instructions provided by the controller. The three kinds of instructions are control, setting and query.

Considering that the oscilloscope has higher sensitivity and its own process control interface, adopt an Agilent DSO5052A oscilloscope to measure the peak-to-peak value of the signal. Choose the LAN process control by adopting an advanced application programming interface, that is, Virtual Instrument Software Architecture (VISA) developed by the National Instrument (NI) Company. After installing the Agilent Input/Output (IO) Libraries Suite, control the machines which are used in experiments by the LAN interface. The software interface is shown in Fig. 2. 


\section{Results AND ANAlyses}

A. Knife

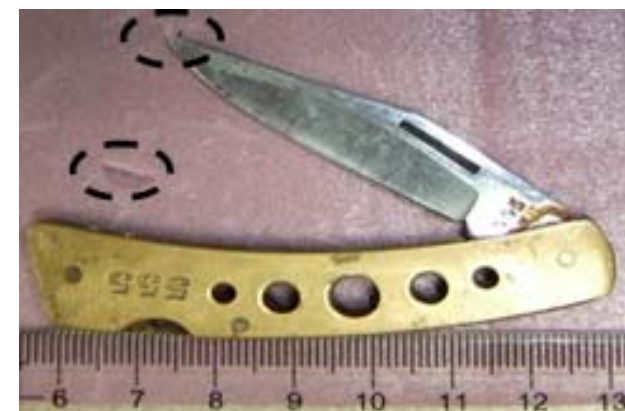

(a)

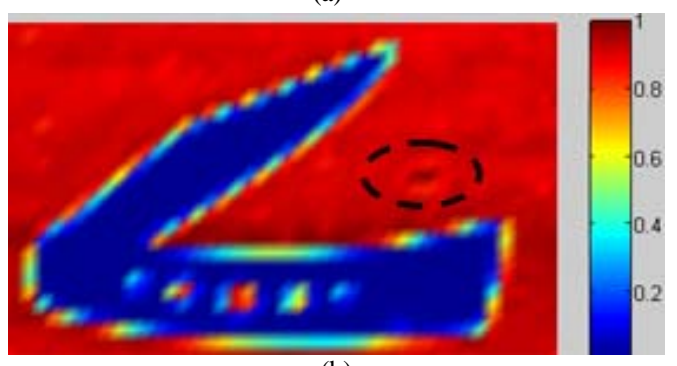

(b)

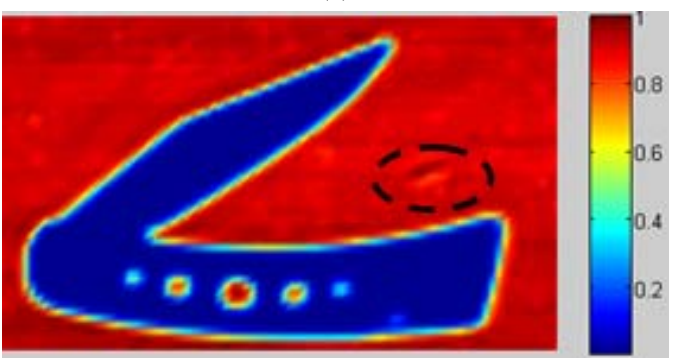

(c)

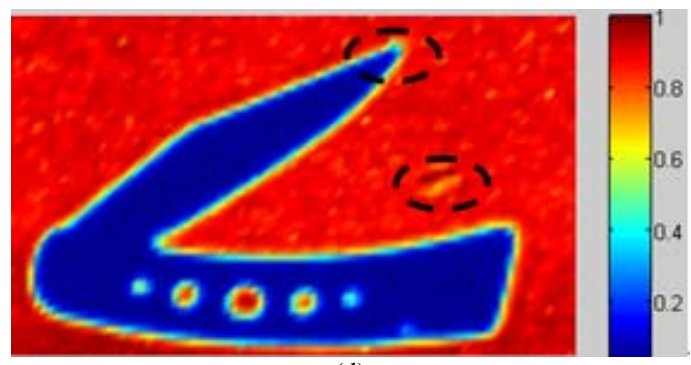

(d)

Figure 3. (a) The optical image of a small knife and foam board. The top dotted elliptical black line indicates a small defect, and the bottom dotted elliptical black line indicates a large defect on the foam board. (b) The transmission image of the knife at $450 \mathrm{GHz}$ with the step interval of $2 \mathrm{~mm}$. (c) The transmission image at $450 \mathrm{GHz}$ with the step interval of $1 \mathrm{~mm}$. (d) The transmission image at $890 \mathrm{GHz}$ with the step interval of $1 \mathrm{~mm}$.

In the first experiment, use a small knife attached to a foam board with thickness of $5 \mathrm{~mm}$, as shown in Fig. 3 (a). The tool holder has five hollow circles, whose maximum diameter is $6 \mathrm{~mm}$. The diameters of other circles on both sides are $4.5 \mathrm{~mm}$ and $3.0 \mathrm{~mm}$ successively. The knife is behind on the back of the foam board, and the foam board is faced to the $\mathrm{THz}$ source in the transmission imaging system (see Fig. 1). Thus the $\mathrm{THz}$ wave passes through the foam board and knife successively, and then arrives at the $\mathrm{THz}$ detector.

Fig. 3 illustrates the transmission $\mathrm{THz}$ images of the knife and foam, in which the spots inside the dotted elliptical black lines in (b)-(d) are corresponding to the defects shown in the dotted elliptical black lines in (a). The phenomena show that the $\mathrm{THz}$ wave cannot pass through the metallic part of the knife, while it can penetrate the foam board easily. Fig. 3(b) and Fig. 3(c) show that the imaging resolution is improved by decreasing the single step interval. Fig. 3(c) and Fig. 3(d) show that the imaging resolution is improved by increasing the incident wave frequency. In Fig. 3 (d), it can be easily distinguished both the small and large defects displayed in 3(a), whereas cannot be easily distinguished in 3(b) and 3(c). Hence, the transmission images verify that the $\mathrm{THz}$ waves can be used to carry out non-destructive flaw detection for materials.

\section{B. Wet Leaf and Dry Leaf}

In the second experiment, two pieces of wet leaf and dry leaf are used to attached to the foam board with thickness of $4 \mathrm{~mm}$, as shown in Fig. 4(a) and Fig. 4(b), respectively. The leaf is behind on the back of foam board and the foam board is faced to the $\mathrm{THz}$ source in the transmission imaging system. The THz images are illustrated in Fig. 4(c) and Fig. 4(d), which show that the water has significant impact on the transmission image. The $\mathrm{THz}$ transmittance will be decreased if the leaf humidity becomes larger. The water is mainly contained in the stem from Fig. 4 (d). Due to the rich of water in stems, the stems look black. The others look transparent because of the lack of water. The black dotted rectangular lines in Fig. 4 (d) are scotch tapes.

\section{CONCLUSION}

In this paper, a compact $\mathrm{THz}$ transmission system for non-destructive imaging is developed by using a BWO source, a Golay-Cell detector, an oscilloscope, and the software. From the given examples, it can be concluded that the $\mathrm{THz}$ imaging properties is relevant to the humidity, thickness, and material properties. The $\mathrm{THz}$ wave cannot penetrate metal (such as a knife). The $\mathrm{THz}$ imaging resolution is restricted by the incident wave frequency. When the incident frequency increases, the imaging resolution becomes better. The $\mathrm{THz}$ imaging resolution is also relevant to the translation step. As the step decreases, the image resolution is improved under the same frequency, while the scanning time will be prolonged. When the translation step is decreased to make the imaging quality be nearly unchanged, there is no need for further reduction. Hence need to select appropriate frequency and translation step to meet the practical imaging requirements. Also other ways, such as digital image processing measures can be used to enhance the image display effects. 


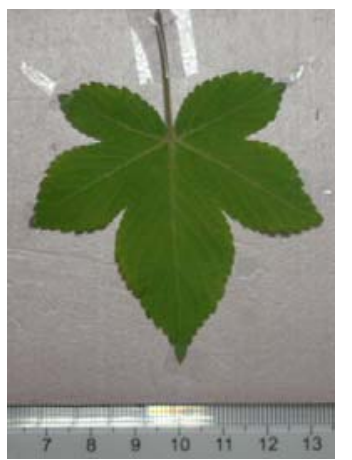

(a)

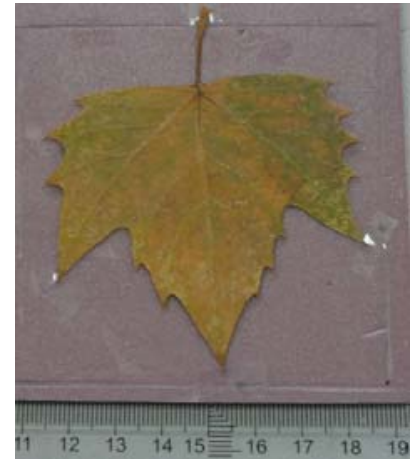

(b)

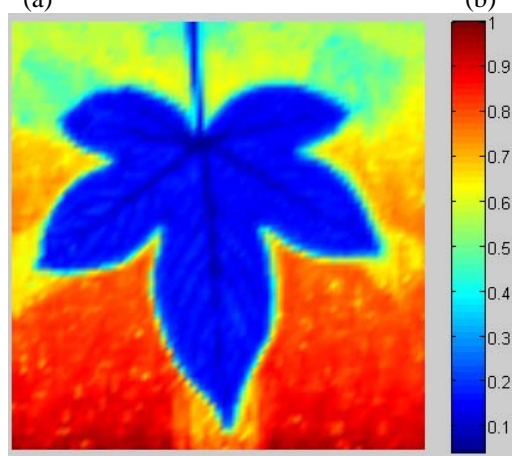

(c)

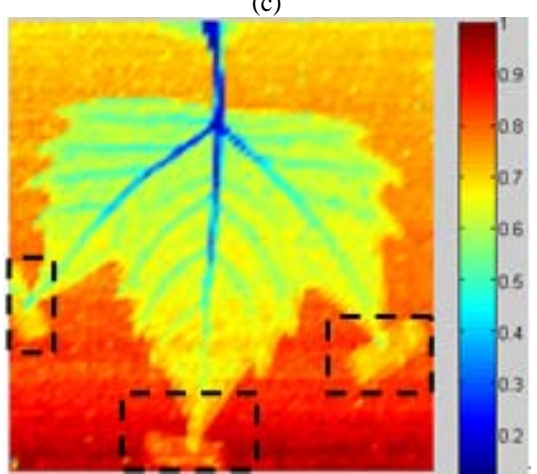

(d)

Figure 4 . (a) The optical image of a piece of wet leaf. (b) The optical image of a piece of dry leaf. (c) The transmission image of the wet leaf at $890 \mathrm{GHz}$ with the step interval of $1 \mathrm{~mm}$. (d) The transmission image of the dry leaf at $890 \mathrm{GHz}$ with the step interval of $1 \mathrm{~mm}$.

\section{REFERENCES}

[1] J. Z. Xu and X. C. Zhang, Terahertz Science Technology and Application. Beijing: Peking University Press, 2007, ch. 1.

[2] P. H. Siegel, “Terahertz technology,” IEEE Trans. Micr. Theory and Tech., vol. 50, no. 3, pp. 910-928, Mar. 2002.

[3] K. B. Cooper, R. J. Dengler, N. Llombart, and P. H. Siegel, "Penetrating 3-D imaging at 4- and 25-m range using a submillimeter-wave radar” IEEE Trans. Micr. Theory and Tech., vol. 56, no. 12, pp. 2771-2778, Dec. 2008.

[4] R. Piesiewicz, C. Jansen, and D. Mittleman, "Scattering analysis for the modeling of THz communication system," IEEE Trans. Antennas Propag., vol. 55, no. 11, pp. 3002-3009, Nov. 2007.

[5] N. Karpowicz, H. Zhong, C. L. Zhang, and X. C. Zhang, "Compact continuous-wave subterahertz system for inspection applications," Appl. Phys. Lett., vol.86, no. 5, pp. 054105-054105-3, Jan. 2005.
[6] R. Appleby and H. B. Wallace, "Standoff Detection of Weapons and Contraband in the $100 \mathrm{GHz}$ to $1 \mathrm{THz}$ Region," IEEE Trans. on Ant. and Prop., vol. 55, pp. 2944-2956, Nov. 2007.

[7] H. Zhong. J. Xu, X. Xie, and X. C. Zhang, "Nondestructive defect identification with terahertz time-of-flight tomography," IEEE J. Sensors., vol. 5, no. 2, pp. 203-208, Apr. 2005.

[8] P. H. Siegel, “THz Technology in Biology and Medicine,” IEEE Trans. Micr. Theory and Tech., vol. 52, no. 10, pp. 2438-2448, Oct. 2004.

[9] B. Hu, X. C. Zhang, and D. Auston, "Free-space radiation from electro-optic crystals,” Appl. Phys. Lett., vol. 56, no. 6, pp. 506-508, Feb. 1990.

[10] Q. Wu, X. C. Zhang, "Free-space electro-optic sampling of terahertz beams,” Appl. Phys. Lett., vol. 67, no. 24, pp. 3523-3525, Dec. 1995.

[11] A. Nahata, J. T. Yardley, and T. F. Heinz, "Two-dimensional imaging of continuous-wave terahertz radiation using electro-optic detection," Appl. Phys. Lett., vol. 81, no. 6, pp. 963-965, Aug. 2002.

[12] B. B. Hu and M. C. Nuss, "Imaging with terahertz waves," Opt. Lett., vol. 20, no. 16, pp.1716-1718, Aug. 1995.

[13] N. Karpowicz, H. Zhong, C. Zhang, K. I. Lin, J. S. Hwang, J. Xu and X. C. Zhang, "Compact continuouswave subterahertz system for inspection applications,” Appl. Phys. Lett., vol. 86, pp. 054105054105-3, Jan.2005.

[14] A. Dobroiu, M. Yamashita, and Y. N. Ohshima, "Terahertz imaging system based on a backward-wave oscillator,” Appl. Opt., vol. 43, no. 30, pp. 5637-5646, Oct. 2004.

[15] K. J. Siebert, H. Quast, R. Leonhardt, "Continuous-wave all optoelectronic terahertz imaging,” Appl. Phys. Lett., vol. 80, no. 16, pp. 3003-3005, Apr. 2002. 